\title{
Signalling for Handover in a Broadband Cellular ATM System
}

\author{
K. Keil, H. Bakker, W. Schödl, M. Litzenburger \\ Alcatel, Corporate Research Center, Stuttgart, Germany \\ e-mail: \{kkeillhbakkerlwschoedllmlitzenb\}@rcs.sel.de
}

\begin{abstract}
This paper presents handover procedures for the Broadband Cellular ATM Access (C-ATM) system which shall provide wireless ATM access for mobile users. Beside the description of the architecture and protocol stack of the system this paper describes message sequence charts for two different handover types: radio and network handover. This differentiation allows most of the handovers to be performed seamlessly, i.e., without loss of ATM cells. This feature is especially important for real time applications such as video.
\end{abstract}

\section{Keywords}

Wireless ATM, Radio Handover, Network Handover, Message Sequence Charts, Connection Admission Control

\section{INTRODUCTION}

In April 1996 the German Federal Ministry of Education, Science, Research and Technology has launched a project called 'Broadband Mobile Communication for Multimedia based on ATM (ATMmobil)'. Within this ATMmobil project several German companies and universities cooperate in the area of a wireless ATM cell transport to end users. Four different system concepts are explored and prototyped within the frame of ATMmobil, one of these is called "Broadband Cellular ATM Access - C-ATM" which aims at providing wireless ATM access in selected areas enabling mobility features within the coverage of the radio cells. After a short system description of the system architecture the handover procedures are described in detail. 


\section{SYSTEM ARCHITECTURE}

The system architecture of the C-ATM system is depicted in Figure 1. It is divided in three parts: mobile terminals, basestation subsystem and fixed network part.

The C-ATM system distinguishes between two types of mobile terminals: a standard ATM terminal connected to a Mobile Terminal Adapter (MTA) and a terminal with radio transceiver, i.e, the MTA functionality is integrated in the ATM terminal. The MTA comprises the radio part and terminates all mobility related protocols, i.e., it hides the mobility aspects from the standard ATM terminal. Both terminal types support the UNI signalling for set up of transparent ATM connections over the air interface.

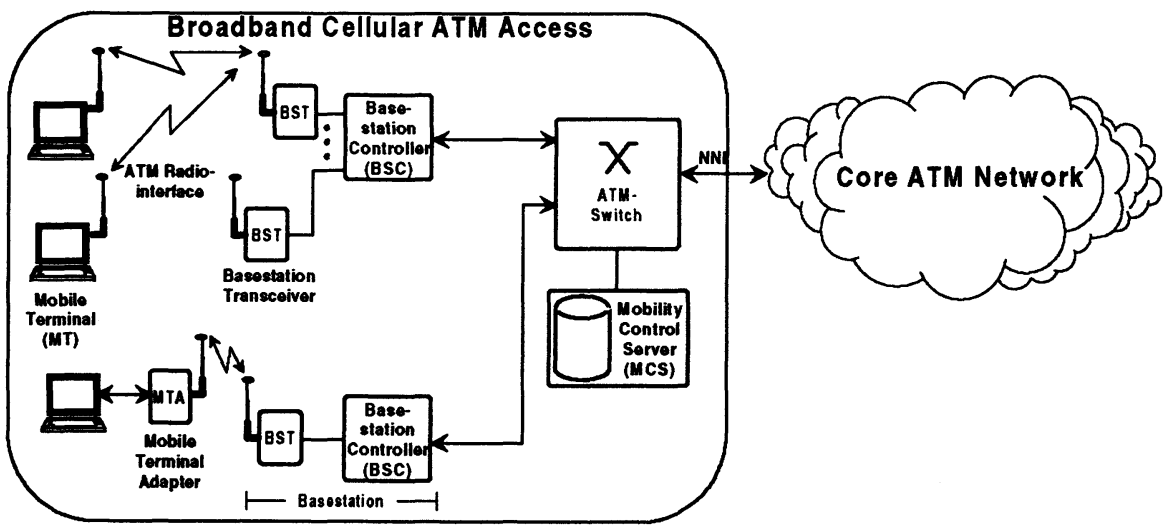

Figure 1: Architecture of the Broadband Cellular ATM access system.

The basestation subsystem consists of Basestation Transceivers (BST) and Basestation Controllers (BSC). A radio cell is set up by a BST which includes baseband processing and the radio frequency part. Several BSTs are controlled by one BSC, i.e. the BSC acts as a multiplexer in the upstream direction towards the fixed network and as a demulitplexer towards the terminal in the downstream direction. As the BST is only working on the physical layer, all mobility related protocols are implemented in the BSC.

The C-ATM system will operate in the $5 \mathrm{GHz}$ frequency range with a bandwidth of about $25 \mathrm{MHz}$ per radio cell in accordance with the (initial) HIPERLAN Type 2 specifications [1]. The maximum peak EIRP (Effective Isotropic Radiated Power) will be $1 \mathrm{~W}$ to cover radio cells with a radius of up to $50 \mathrm{~m}$, which leads to a picocellular environment. Furthermore, it is intended to use OFDM (Orthogonal Frequency Division Multiplexing) as modulation scheme.

In our envisaged C-ATM system the fixed network part comprises as central part an ATM switch which connects the basestation subsystem via an NNI interface to 
the core ATM network. Connections to other C-ATM islands are not considered here. The mobility functions such as Location Management, extended Connection Admission Control (CAC), and Handover management are not handled by the ATM switch, thus a Mobility Control Server (MCS), which is connected to the switch via an ATM interface, is used to support the ATM switch. The introduction of a MCS requires only minor modifications within the Call Control (CC) unit of a standard ATM switch. Both the ATM switch and the MCS are acting towards the core ATM network and towards the radio access system as a mobility enhanced ATM switch. Within a demonstrator platform which will be prototyped by the C-ATM project the $\mathrm{CC}$ unit will also be implemented within the MCS as a second task. Following this approach the modifications of the CC which become necessary to support mobility functions (e.g. handover), can easily be implemented. Thus, all UNI messages of the CC protocol (setup, connect, release) are terminated in the MCS and not in the ATM cell site switch.

Figure 2 shows the user plane protocol stack of the C-ATM system. Providing wireless access the user of the C-ATM system should not be aware of the transmission of ATM cells over the air interface, i.e., the transmission should be fully transparent without any degradation compared to fixed access.

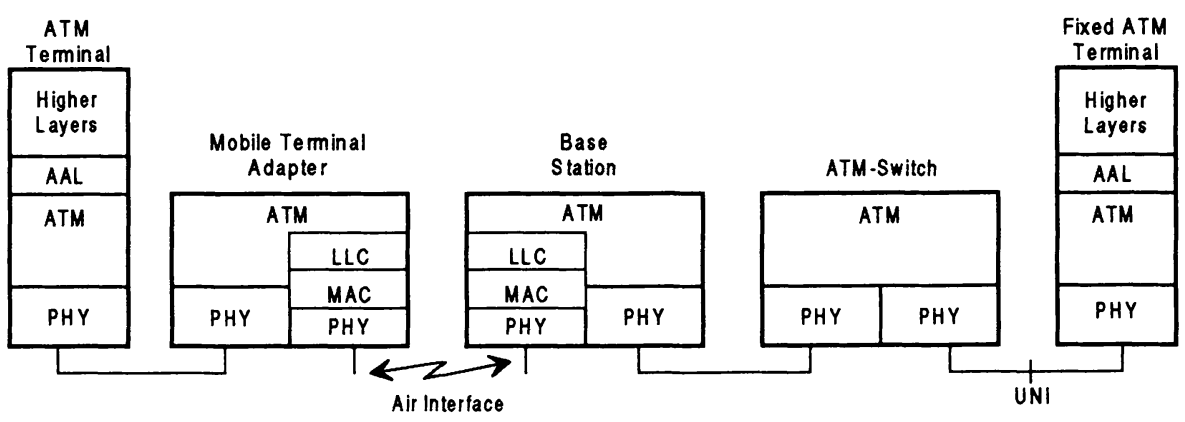

Figure 2: Protocol stack user plane.

To hide the specific air interface characteristics from the ATM layer, an additional Data Link Control (DLC) layer has been introduced. The DLC layer consists of a Media Access Control (MAC) and a Logical Link Control (LLC). While the MAC protocol enables the statistical multiplexing of multiple mobile terminals on the air interface, the LLC protocol improves the ATM cell error rate by a controlled retransmission of corrupted ATM cells. The retransmission will become necessary as soon as the Forward Error Correction - (FEC-) mechanism implemented in the physical layers fails. All other layers are unmodified, enabling a transparent end-to-end ATM Adaptation Layer (AAL) connection between a wireless and a second fixed/wireless ATM terminal. The protocol stack for the control plane is depicted in Figure 3. The functions for Mobility Control 
(MC) and Call Control (CC) are handled by the CC/MC layer of the MTA, the BSC and the ATM switch / Mobility Control Server. Within the ATM terminal and the fixed ATM terminal representing the fixed ATM network part, no MC is implemented. Mobility Control messages are exchanges between the MTA / BSC and the Mobility Control Server, which controls the mobility functions mentioned above.

Below the CC/MC protocol layer in a new network layer protocol is introduced, the so-called Signalling Network Layer (SNL) [2]. The main function of the SNL protocol is to deliver signalling messages to the corresponding signalling end point, even to a mobile end point. The SNL hides the fact that the mobile terminals move within the access network from the application layer, i.e. $\mathrm{CC} / \mathrm{MC}$.

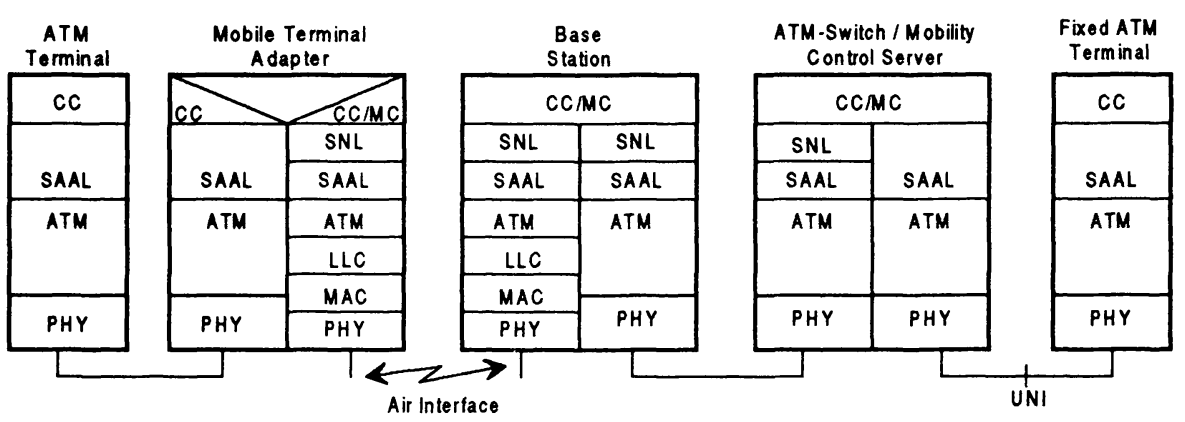

Figure 3: Protocol stack control plane.

\subsection{Handover in a C - ATM System}

A key function within any mobile cellular communication system is handover, which allows to guarantee a certain requested quality of service for the connections of a mobile user while moving. A handover may also become necessary for reasons of traffic load redistribution among radio cells. This section sketches the mechanisms and signalling procedures for handover in a wireless cellular ATM network.

Most of these procedures are included in the baseline text for wireless ATM specifications of the Wireless ATM working group of the ATM Forum [6].

\subsection{Definitions and Functional Description}

Based on the architecture of the C-ATM system (Figure 1), we distinguish between two types of handover which are depicted in Figure 4 [3]. 
- Radio handover: The mobile terminal moves from the coverage area of one BST to the coverage area of another BST, but stays within the area of one BSC.

- Network handover: The mobile terminal changes its location from the area of one BSC to the area of another BSC.

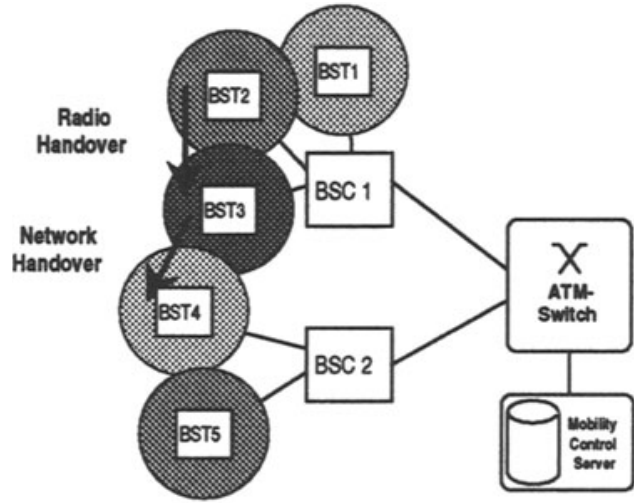

BSCi: Basestaion Controller i

BST j: Basestaion Transcoiver $j$

Figure 4: C-ATM handover types.

Since radio handovers might occur quite frequently in a pico-cellular environment, it is important that a radio handover can be performed seamlessly, i.e., without loss of data and with minimum additional delay. The ATM switch is not involved in the handover execution. The synchronisation of the ATM cells is performed within the DLC layer and is described in [3].

For a network handover, on the other hand, the output port of the ATM switch will be changed in order to handover a mobile terminal's current connections to another BSC, i.e., the path has to be modified. In contrast to a path extension scheme (cf., e.g., [4]), path rerouting requires no switching functionality within the BSCs. But with the ATM switches currently available, ATM cells might be lost, because it is not possible to synchronise ATM cell streams arriving at different switch ports. Therefore, the network handover will not be seamless.

Concepts for a seamless network handover [7] are currently not discussed in detail within the Wireless ATM working group of the ATM Forum.

The C-ATM handover procedures which combine principles of DECT and GSM have the following properties:

- The handover will be mobile-initiated, i.e., the mobile terminal decides when to request a handover. Together with the request, it shall provide a sorted list of BSC/BST identifiers and radio-link quality measurements of the neighbouring radio-cells. This request is forwarded to the MCS. 
- The decision, whether to accept the handover request and which target radio cell to choose, will be performed in the MCS, taking into account the link qualities of the possible target cells and the current traffic load distribution. Therefore, the MCS has to monitor the traffic load in all attached radio cells. An extended Connection Admission Control (CAC) has to be implemented which checks whether the required capacity is available in the new radio cell. The radio handover execution takes place within the BSC of the two radio-cells involved, while for the execution of a network handover additionally the ATM switch is required for the rerouting of cells

In the following sections the signalling flows for both C-ATM handover types are described. The algorithms to obtain measurement values for the radio-link quality of the current connection and the neighbouring radio cells are not included. These algorithms are necessary to decide, when a handover should be requested which have to be implemented in the mobile terminal.

\section{SIGNALLING FLOW FOR HANDOVER PROCEDURES}

\subsection{Definitions and Assumption}

In the following we assume the Mobile Terminal is composed of a standard terminal and a MTA for termination of the radio link - ranging from the physical layer up to the MC function (see Figure 3). So the MTA is the endpoint for all the mobility functions.

Within our demonstration platform we will use fixed separate mobility control channels established between MTA and BSC, MTA and MCS and furthermore between BSC and MSC for the exchange of mobility control message between those entities. Within the C-ATM system concept a more advanced solution based on SNL principles is envisaged [5].

The mobility control messages for any handover procedure to and from the MTAMC instance are exchanged via the current active radio link (backward handover).

The MTA-MC instance is informed about the session (i.e. number of virtual connections, VPI/VCI and capacity per virtual connection ) currently active on the terminal either by the $\mathrm{CC}$ resident within the terminal or by the mobility control (MC) instance of the MCS.

Furthermore, as addressed above, two types of handover have been defined. The decision which handover type shall be executed will be made by the MCS, taking into account the availability of capacity within the proposed target radio cells. In cases, where both types are possible, priority is given to radio handover versus network handover, because it performs a seamless transition to another radio cell. The decision to initiate a network handover is based on two criteria: 
- The list received from the mobile station does not include an adjacent radio cell belonging to the same BSC, or

- there is no capacity available in an adjacent radio cell belonging to the same BSC.

Concerning the availability of capacity, the C -ATM project has classified handover procedures as follows:

- A normal handover procedure, e.g. normal radio / network handover procedure if enough capacity for the requested handover is available in one or more target cells.

- A restricted handover procedure, e.g. restricted radio / network handover procedure if in none of the proposed target cells the required capacity is available for the handover of a session with multiple active virtual connections. Then the MCS checks if a release of one or more virtual connection(s) of the current session (i.e. reduction of capacity requirement) makes a handover possible. If this is the case the MCS then initiates via the CC Function and based on a standard signalling procedure the release of the defined virtual connection(s) before starting the handover procedure.

In case the required capacity is not available within the proposed target radio cells (neither for the requested handover with only one active virtual connection, nor in case of restriction for a session with multiple active virtual connection) the MCS rejects the handover request from the MTA.

\subsection{Handover Procedures}

A software process within the MTA responsible for monitoring the transmission quality over the radio link will initiate a handover procedure by informing the MTA-MC instance if the received signal strength falls short of a defined threshold limit. The MTA-MC instance will inform its counterpart in the Mobility Control Server (MCS) by sending a HO_Request_Indication message (Figure 5), which includes among others the following parameters: a sorted list with the BSC/BST combinations. Furthermore the MSC-MC starts the timer T1, which is introduced to monitor the reaction of the MCS-MC instance and the stability of the radio link. In case of time-out of T1, the MTA-MC instance shall start a forward handover procedure.

On receipt of the HO_Request_Indication message the MCS-MC instance assigns a process identity (HO_ID) and checks, based on the BSC/BST combinations received, whether enough capacity for a handover is available in the proposed target cells. Depending on the result of this extended Connection Admission Control (CAC), the MSC-MC instance will define the handover type and the condition for the handover procedure (i.e. normal or restricted) or will reject the handover request if the required bandwidth capacity is not available. 


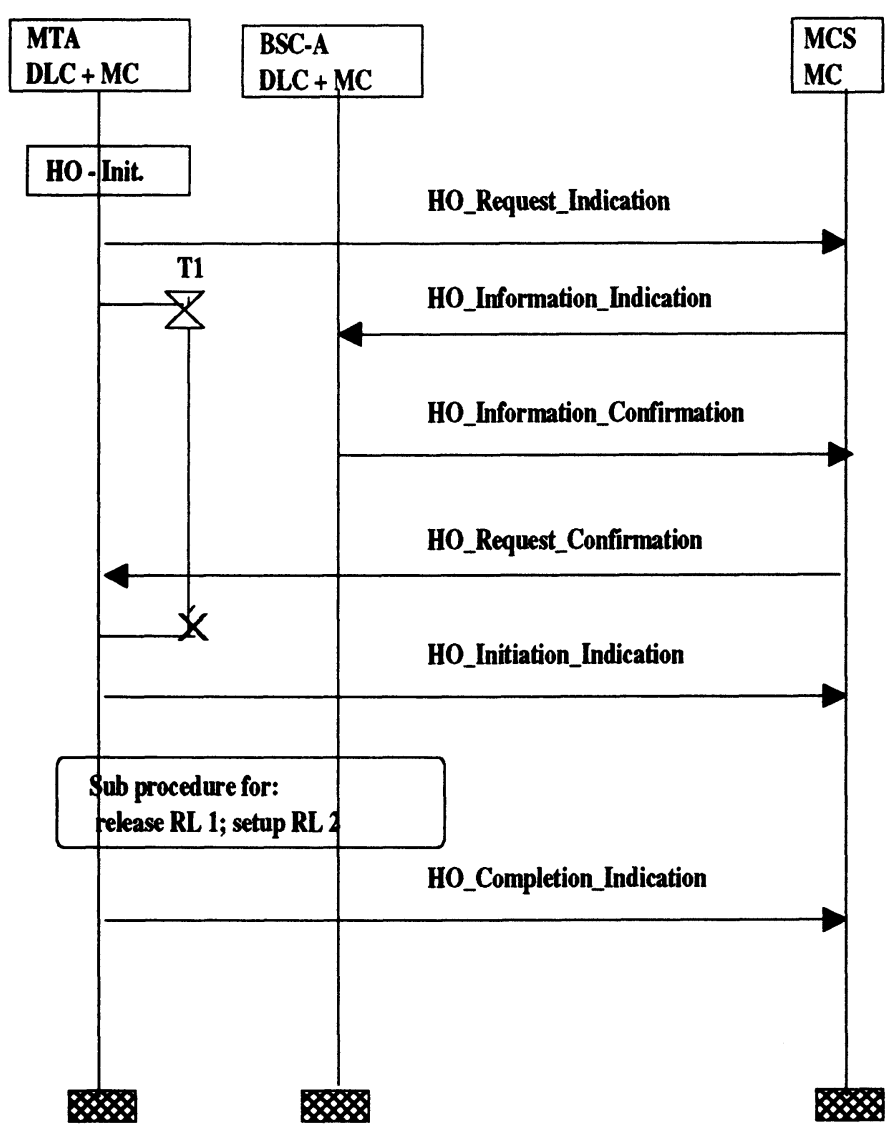

Figure 5: Message Sequence Chart for a normal radio handover procedure.

In the section below, the message sequence flow for each particular case is described in detail.

\subsubsection{Normal Radio Handover Procedure}

In this case the adjacent radio cell belonging to the same BSC provides enough capacity and the MCS-MC instance defines the corresponding BSC_ID / BST_ID combination for the radio handover and sends this combination with a HO_Information_Indication message to the MC instance of the BSC.

On receipt of the HO_Information_Indication message the BSC-MC instance informs the DLC process to prepare itself for a handover to a transceiver defined by the received parameters. After completion of the preparation phase the BSCMC instance sends the HO_Information_Confirmation message to the MSC-MC instance to indicate that the BSC-MC and BSC-DLC instances are prepared for the handover. 
To inform the MTA-MC about the target cell and the handover type, the MSCMC instance sends the HO_Request_Confirmation message to the MTA-MC, which includes, among others, an identifier for the handover type (HO-Flag) and the identifier of the new selected radio cell.

On receipt of the confirmation the MTA-MC instance clears timer T1 and checks the selected handover type. For radio handover, the MTA-MC instance sends a HO_Initiation_Indication message to the MSC-MC instance to indicate that the change of the radio links will be started. Then the MTA stops the transmission of ATM cells over the current radio link and establishes a new radio link to the new BST.

If the new radio link is set up, both DLC instances restart the transmission of ATM cells send a confirmation about the completion to their respective MC instances.

On receipt of the confirmation message from the DLC instance, the MTA-MC instance sends a HO_Completion_Indication message to the MCS-MC instance and terminates the handover procedure within the MTA.

On receipt of the HO_Completion_Indication message the MCS-MC instance will release the assigned HO_ID, and will update the database of the MCS, e.g., mobile terminal located in a new location area.

\subsubsection{Normal Network Handover Procedure}

In cases, where only BSC/BST combinations from a neighbour BSC are proposed, or a radio handover is not possible due to capacity limitations but enough capacity is available in radio cells from a neighbour BSC, the MCS-MC instance decides to perform a network handover.

Compared to the radio handover procedure, additional messages are necessary for a network handover due to the fact that the involved radio cells belong to two different BSCs. In order to inform the MC instances of both BSCs about the planned handover, the MCS-MC instance sends a HO_Release_Indication message to BSC-A and a HO_Information_Indication message to BSC-B (see Figure 6).

As described in the message sequence flow for the radio handover procedure (Figure 5), the MC instances of BCS-A and BSC-B inform and request their respective DLC instances to get prepared for the handover procedure. Then both $M C$ instances sends a relevant acknowlegde message to the MCS-MC instance.

On receipt of the HO_Request_Confirmation message the MTA-MC checks the HO_Flag parameter and handover type, the MTA-MC instance requests from its MTA_DLC to stop transmission of ATM cells.

The MTA-MC then sends the HO_Initiation_Request message to the MCS-MC instance indicating to be ready for the rerouting procedure.

On receipt of the HO_Initiation _Request message the MCS-MC instance requests from the Bearer Control (BC) instance inside the MCS, to reroute the path through the ATM switch for the involved virtual connection(s) and the associated signalling channel by sending a $R+S \_$Request message. 
The BC instance modifies the routing tables of the ATM switch so that the ATM cells of the defined virtual connection(s) will be routed to the port where the new BSC is connected. The new BSC, already prepared by the HO_Informaton_Indication message, will store the ATM cells until the new radio link to the MTA has been established.

After termination of the rerouting procedure the $\mathrm{BC}$ instance sends the $\mathrm{R}+\mathrm{S}$ _Confirmation message to the MSC-MC instance as an indication.

On receipt of the $\mathrm{R}+\mathrm{S}$ _Confirmation message the MSC-MC instance informs the MTA-MC instance by means of the HO_Initiation_Confirmation message that the path through the switch for user data and associated signalling channel has been changed and the change for the radio link can be started. The MTA-MC instance then request from the MTA-DLC instance to change the radio links. The release of the radio link L1 and the setup of the new radio link L2 is achieved via exchange of DLC protocol messages between the MTA-DLC and the DLC instances of BSC-A and BSC-B.

After the change of the radio links is completed the MTA-DLC instance sends a confirm message to the MTA-MC instance.

To inform the MSC-MC instance about the end of the handover procedure within the MTA, the MTA-MC instance then sends the HO_Completion_Indication message. This message is transferred in upstream direction over the new established radio link using the Basestation proprietary mobility control channel, while for the mobility channel in downstream direction to the MTA a rerouting is still required.

Therefore, on receipt of the HO_Completion_Indication message the MCS-MC instance contacts again the $B C$ instance and request by the $R+S \_$request message to change for the downstream mobility channel the path through the ATM switch. The BC instance informs the MSC-MC instance about the termination of the rerouting procedure by sending the $\mathrm{R}+\mathrm{S}$ _Confirmation message. Using the new downstream path, the MSC-MC instance sends the HO_Completion_Confirmation message to the MTA-MC instance to indicate the termination of the handover procedure on the MSC side.

The MSC-MC instance then releases the assigned HO_ID and updates its own database and other databases relevant for mobility management inside the MCS.

\subsubsection{Restricted Handover Procedure}

If in none of the proposed target cells the required capacity is available for the handover request from a session with multiple virtual connections, the MSC-MC instance checks and decides which virtual connections shall be released, before a handover procedure can be performed. Thus, for both handover types, the MSC$\mathrm{MC}$ instance will contact the CC instance and request the release of the defined virtual connections, before the handover procedure can proceed.

Based on a standard signalling procedure the $\mathrm{CC}$ instance performs the release of the defined virtual connection(s) and confirms the release to the MSC -MC instance. 


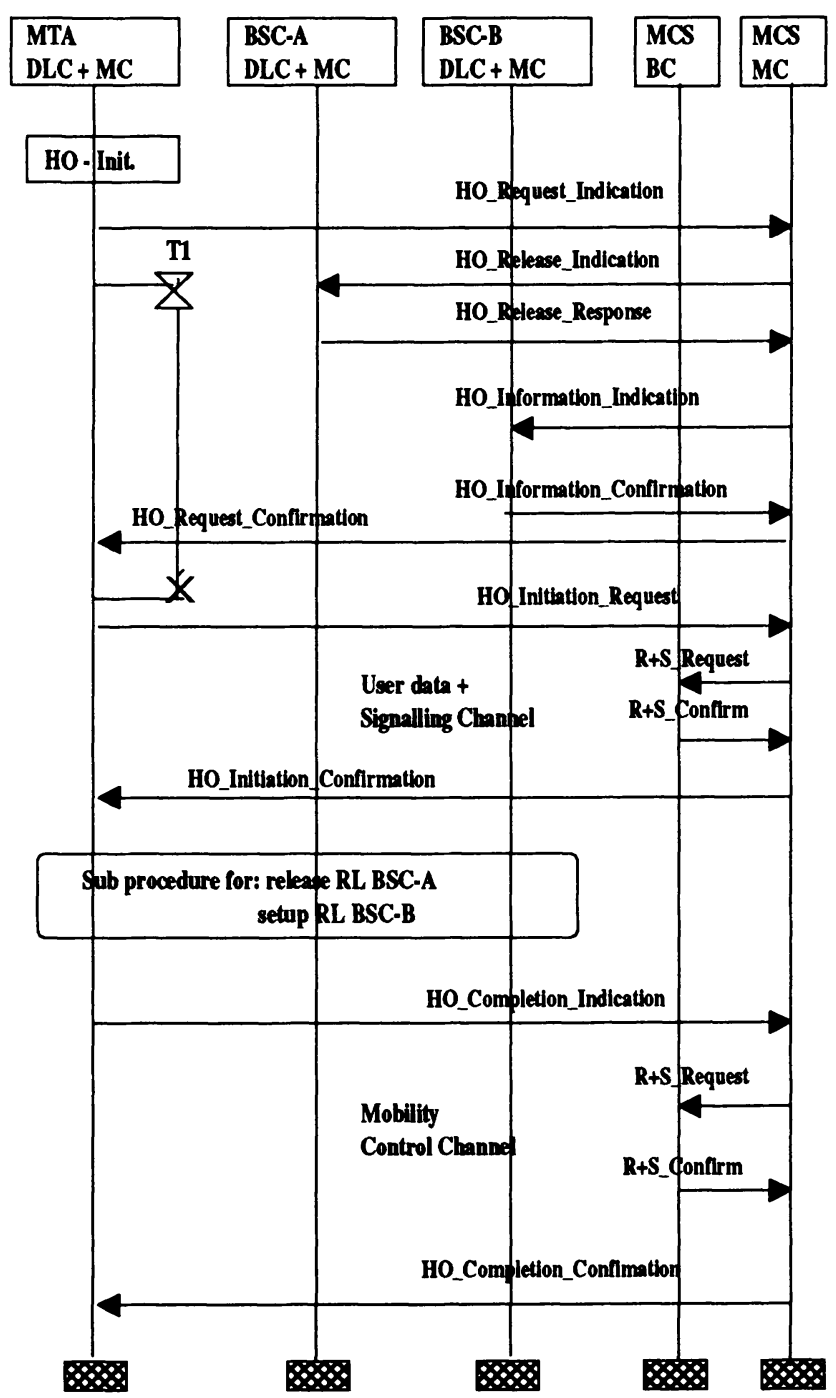

Figure 6: Message Sequence Chart for a normal network handover procedure.

On receipt of the confirmation message, the MCS-MC instance defines the BSC/BST combination for the target cell and the handover type, which determines the further operations for the handover procedure.

\subsubsection{Aborted Handover procedure}

In cases where not enough capacity is available in any of the proposed target radio cells, the MSC-MC instance rejects the HO_Request_Indication message by means of the HO_Request_Rejection message which leads the MTA-MC instance to abort the handover procedure. 


\section{SUMMARY}

This contribution describes the handover procedures which will be used in the Broadband Cellular ATM Access project. After outlining the system architecture two different types of handover have been introduced: the radio handover and the network handover. These handover types are reflected in the corresponding simplified message sequence charts showing the network entities involved. Further work has to be performed to extend the standard ATM Connection Admission Control (CAC). While in fixed ATM networks the CAC function is only used during the connection setup, in C-ATM systems the CAC function has also to be used during each handover to a new radio cell.

\section{ACKNOWLEDGEMENT}

The work presented in this paper has been supported by the German Federal Ministry of Education, Science, Research, and Technology.

\section{REFERENCES}

[1] ETSI RES10 Technical Report "High Performance Radio Local Area Networks (HIPERLANs), Requirements and Architecture," Draft, Sophia Antipolis, 1996.

[2] Mitts, H.; Luijten, G.; Korinthios, J.A.; Nelson, J.: Connectionless signalling network layer in UMTS, IEEE Personal Communications Magazine, June 1996.

[3] H. Bakker, W. Schödl: ATM up to the Mobile Terminal- Impact on the Design of a Cellular Broadband System, ISS'97: XVI World Telecommunications Congress, Toronto, 21.09. - 26.09.97, pp. 379 - 385

[4] B. Walke, D. Petras, D. Plassmann: "Wireless ATM: Air Interface and Network Protocols of the Mobile Broadband System," IEEE Personal Communications: Wireless ATM, Vol. 3, No. 4, August 1996, pp. 50 - 56.

[5] H. Bakker, W. Schödl, M. Litzenburger, K. Degenhard: A Channel Concept for Signalling Messages in a Wireless ATM Access Network with Server Based Control, ACTS Mobile Summit 1997, Aalborg, Denmark, October 1997, pp. 441 - 446

[6] ATM Forum: Wireless ATM working group , Baseline Text for Wireless ATM specifications, ATM Forum BTD-WATM-01.05, December 1997

[7] H. Mitts, H. Hansén, J. Immonen: Lossless handover for wireless ATM, Mobicom'96, Proceedings, pp. 85-96, New York, U.S., 10 -12 Nov 96 